\title{
Diabetes: tendinites e entesopatias $\left.{ }^{*}\right)$
}

\section{Diabetes: tendinitis and enthesophathy}

\author{
Ludmilla Daru Rey ${ }^{(1)}$, Rocio Della Coletta ${ }^{(2)}$, Maira MuKai ${ }^{(3)}$, Marília B Silva ${ }^{(4)}$, Thelma L Skare ${ }^{(5)}$
}

\section{RESUMO}

Objetivo: Verificar a prevalência de tendinites e/ou entesopatia em pacientes portadores de diabetes mellitus (DM) tipos 1 e 2 e relacionar a presença das mesmas com a idade dos pacientes, tipo de diabetes e níveis de glicemia. Métodos: Foram estudados 149 pacientes com DM (64 homens e 85 mulheres), sendo 12 portadores de DM tipo 1 e 137 de DM tipo 2, e 55 pessoas normais (23 homens e 32 mulheres). Os indivíduos foram submetidos a exame fisico para tendinites e entesopatias, dosagem de glicemia de jejum e hemoglobina ( $\mathrm{Hb}$ ) A1C. Resultados: Achou-se tendinite e/ou entesopatia em 3,6\% do grupo-controle contra 32,21\% nos diabéticos. A freqüência de tendinites e/ou entesopatia no diabético era semelhante nos dois sexos, e não se notou correlação entre a presença dessas e a idade, o tempo de doença e o tipo de DM. No que diz respeito a níveis glicêmicos, apesar de os diabéticos com tendinites apresentarem uma média das glicemias levemente superior à dos sem tendinites, o estudo não demonstrou diferença estatisticamente significativa, acontecendo o mesmo em relação à Hb A1C. Conclusões: Concluiu-se que pacientes diabéticos apresentam uma maior prevalência de tendinites e/ou entesopatias que a população normal e que estas não dependem da duração da doença nem do tipo de diabetes. A glicemia de jejum e $\mathrm{Hb}$ A1C não refletem adequadamente as alterações no metabolismo do colágeno, responsáveis pelas entesopatias e tendinites nesses pacientes.

Palavras-chave: diabetes mellitus, entesopatias, tendinites.

\section{INTRODUÇÃO}

Pacientes diabéticos são aquinhoados com uma série de complicações para o aparelho musculoesquelético, tais como osteopenia, artropatias de Charcot, síndromes do túnel do carpo, periartrites do ombro, dedos em gatilho, síndrome da mão rígida, contratura de Dupuytren etc ${ }^{(1)}$.

\begin{abstract}
Objective: To verify the prevalence of tendinitis and/or enthesopathy in diabetic patients and its relationship to with age, type and duration of diabetes as well as glycemic control. Methods: We studied 55 normal controls (23 males and 32 females) and 149 diabetic patients (64 males and 85 females); 12 of them with type 1 diabetes and 137 with type 2. We performed physical examination for tendinitis and determination of fasting glycemic levels and hemoglobin $(\mathrm{Hb})$ A1C. Results: We found that $3.6 \%(n=2)$ of normal persons, and $32.31 \%$ of diabetic patients $(n=48)$ had tendinitis and/or enthesopathy. The frequency of tendinitis and/or enthesopathy in diabetic patients was similar in both gender and we could not find a relationship with age, disease duration and diabetes type. Concerning glycemic control, we found that patients with tendinitis had a glycemic medium level higher than those without it, but no significant statistical difference could be demonstrated, happening the same with HbA1C. Conclusions: We concluded that diabetic patients have a high prevalence of tendinitis and/or enthesopathy, which does not depend on disease duration or diabetes type. Fasting glycemia and HbA1C do not reflect collagen metabolism changes that favor enthesophaties and tendinitis in these patients.
\end{abstract}

Keywords: diabetes mellitus, tendinitis, enthesopathy.

Algumas dessas patologias, como as tendinites, entesopatias e as síndromes de mobilidade articular reduzida, parecem ter um processo etiopatogenético comum, estando associadas a alterações pós-sintéticas no colágeno de estruturas periarticulares ${ }^{(2)}$. Acredita-se que essas alterações se devam à hiperglicemia crônica e são semelhantes àquelas que acontecem no processo de envelhecimento. Nessas duas

\footnotetext{
* Disciplina de Reumatologia da Escola de Medicina da Faculdade Evangélica do Paraná. Recebido em 19/4/2002. Aprovado, após revisão, em 23/1 1/2002.

1. R2 de Clínica Médica do Hospital Evangélico de Curitiba (HUEC), PR.

2. R2 de Clínica Médica do HUEC.

3. R1 de Clínica Médica do HUEC.

4. Professora Assistente da Disciplina de Reumatologia da Escola de Medicina da Faculdade Evangélica do PR.

5. Professora Assistente da Disciplina de Reumatologia da Escola de Medicina da Faculdade Evangélica do PR.

Endereço para correspondência: Thelma L Skare. Rua João Alencar Guimarães, 796 CEP 80310-420, Curitiba, PR, e-mail: tskare@onda.com.br
} 
situações, encontra-se um aumento nas ligações intermoleculares do colágeno, o qual passa a apresentar uma cor mais escurecida (amarronzada), aumento na fluorescência, resistência à digestão enzimática e perda da elasticidade ${ }^{(2)}$.

Este estudo foi feito com o propósito de conhecer a prevalência de tendinites e/ou entesopatias na nossa população diabética e de tentar correlacionar a ocorrência das mesmas com idade dos pacientes, tipo de diabetes mellitus (DM) e com o controle glicêmico.

\section{PACIENTES E MÉTODOS}

Foram estudados 149 pacientes diabéticos, sendo 64 homens e 85 mulheres, com idade variando de 3 anos a 93 anos (média de 58,99; DP $= \pm 13,55$ ) e tempo de diagnóstico de DM entre 1 mês e 33 anos (média de 7,22 anos; DP $= \pm 6,26)$. Doze pacientes $(8,05 \%)$ eram portadores de DM tipo 1 e 137 (ou 91,95\%) eram portadores de DM tipo 2. Cerca de 19 pacientes $(12,75 \%)$ faziam controle apenas com dieta; 77 pacientes $(51,68 \%)$ utilizavam isoladamente hipoglicemiantes orais e 53 pacientes $(35,57 \%)$ faziam uso de insulina isolada ou associada a hipoglicemiantes orais. Neste grupo, $47 \%$ dos pacientes submetiam-se a tarefas com esforço repetitivo e/ou trabalho braçal.

Para o grupo-controle foram estudadas 55 pessoas não diabéticas, sendo 23 do sexo masculino e 32 do sexo feminino, com idades entre 6 anos e 88 anos (média de $47,13$; DP $= \pm 19,04)$. No grupo-controle, $44 \%$ dos componentes exerciam tarefas com esforço repetitivo e/ou trabalho braçal. Todos foram submetidos a anamnese $\mathrm{e}$ exame físico para diagnóstico de tendinites de supra-espinhosos, bíceps, epicondilites dos cotovelos, tenossinovite de De Quervain e tendinite de tendão de Aquiles. $\mathrm{Na}$ anamnese foram consideradas apenas as queixas atuais. Os casos positivos tiveram seu diagnóstico clínico confirmado pelas manobras de Yergason (para tendinite bicipital), do arco doloroso (para tendinite do supra-espinhoso) e de Finkelstein (para tendinite de De Quervain). O exame físico e a execução das manobras obedeceram à descrição feita na literatura específica ${ }^{(3)}$. Todos foram submetidos a dosagens de glicemia de jejum, e os diabéticos foram, também, submetidos à dosagem de $\mathrm{Hb} \mathrm{A1C}$. A dosagem da primeira foi feita pelo método enzimático colorimétrico e a da segunda, por turbidimetria, usandose, para ambas, o aparelho Cobas Mira Plus.

Do ponto de vista estatístico, foi realizado, inicialmente, um estudo descritivo com objetivo de se conhecer as características relacionadas à distribuição de dados, sua tendência central e variabilidade, presença de valores discrepantes e verificação de suposição de normalidade. Nesta etapa foram construídos gráficos do tipo box-plot para detecção de valores considerados discrepantes. Em seguida, foram realizados testes estatísticos não paramétricos de Qui-quadrado, exato de Fisher e Mann-Whitney. O nível de significância adotado foi de 5\%.

\section{RESULTADOS}

Dos pacientes diabéticos estudados, $101(67,78 \%)$ não possuíam tendinites e/ou entesopatias e 48 (32,21\%) tinham um ou mais pontos de envolvimento. No grupo-controle, apenas $2(3,6 \%)$ eram portadores de tendinites, o que mostrou que a prevalência de tendinites e entesopatias é significativamente maior nos diabéticos $(p=0,0002)$.

Dos pacientes diabéticos portadores de entesopatia e/ou tendinite, 21 (43,75\%) eram homens e 27 (56,25\%) eram mulheres. Estudando-se a porcentagem de indivíduos diabéticos com achados positivos, verificou-se que 32,81\% dos homens e $31,76 \%$ das mulheres eram portadores de um ou mais pontos de tendinites e/ou entesopatias (Figura 1).

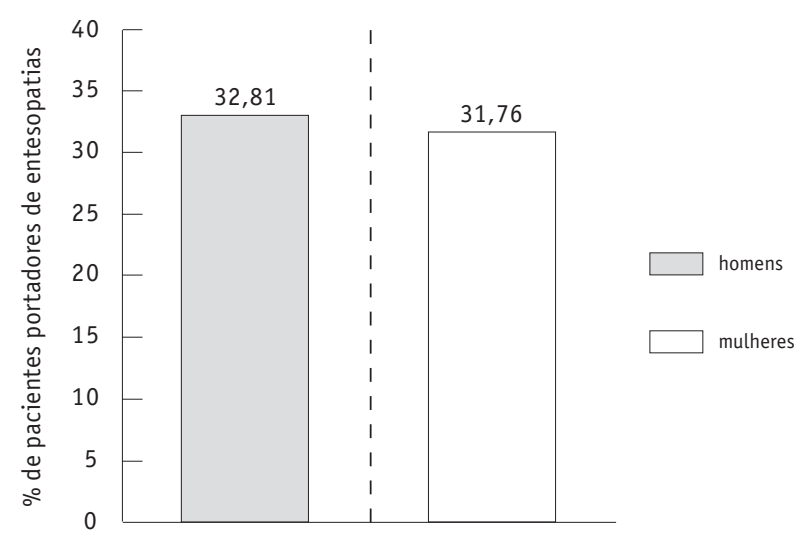

Figura 1 - Prevalência de entesopatia em pacientes com diabetes de acordo com o sexo.

Nos 47 pacientes com achados positivos, foram verificados 96 pontos dolorosos, assim distribuídos: 27 no tendão do supra-espinhoso, 25 no tendão bicipital, 14 no epicôndilo lateral e 13 no epicôndilo medial dos cotovelos, 14 no tendão de Aquiles e 3 no tenossinovite de De Quervain. Dessa maneira, o ombro foi a estrutura mais envolvida $(54,16 \%)$, seguido pelo cotovelo $(28,12 \%)$, tornozelos $(14,58 \%)$ e mãos $(3,12 \%)$ (Figura 2$)$. 


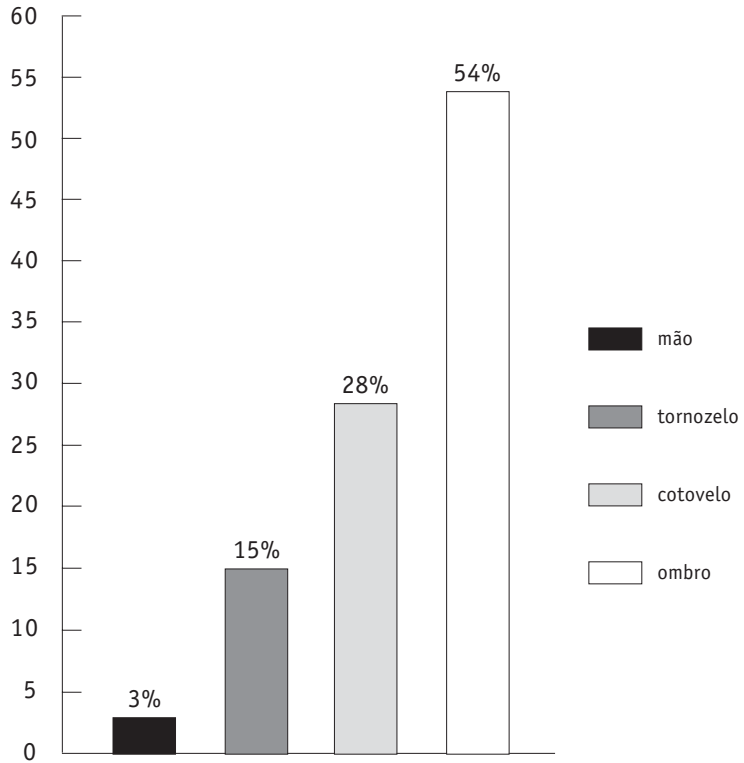

Figura 2 - Padrão envolvimento entesopático na população diabética.

Os valores para glicemia de jejum na população-controle variaram entre $50 \mathrm{mg} / \mathrm{dl}$ e $107 \mathrm{mg} / \mathrm{dl}$ (média de 86,6; $\mathrm{DP}= \pm 12,83$ ). Os valores encontrados para glicemia de jejum e os da $\mathrm{Hb} \mathrm{A} 1 \mathrm{C}$ encontram-se relacionados respectivamente nas Tabelas 1 e 2 .

O comportamento da glicemia de jejum e da $\mathrm{Hb}$ A1C nos pacientes com e sem tendinites estão ilustrados pelas Figuras 3 e 4.

TABELA 1

TAXA DE GLICEMIA EM JEJUM DA POPULAČ̃̃ DIABÉTICA ESTUDADA

\begin{tabular}{lcc}
\hline Glicemia $(\mathrm{mg} / \mathrm{dl})$ & Freqüência & $\%$ \\
\hline Até 110 & 21 & 14,38 \\
$111-210$ & 79 & 54,11 \\
Acima de 210 & 46 & 31,51 \\
\hline
\end{tabular}

* Sem informação: 3 pacientes

TABELA 2

TAXa de hemoglobina $A_{1}$ NA POPULAÇÃO DIABÉTICA ESTUDADA

\begin{tabular}{lcc}
\hline Hemoglobina $\mathbf{A}_{1 \mathrm{C}}(\%)$ & Freqüência & $\%$ \\
\hline Abaixo de 7 & 12 & 8,11 \\
$7-8,3$ & 48 & 32,43 \\
$8,4-9,0$ & 20 & 13,51 \\
Acima de 9,0 & 68 & 45,95 \\
\hline
\end{tabular}

* Sem informação: 1 paciente

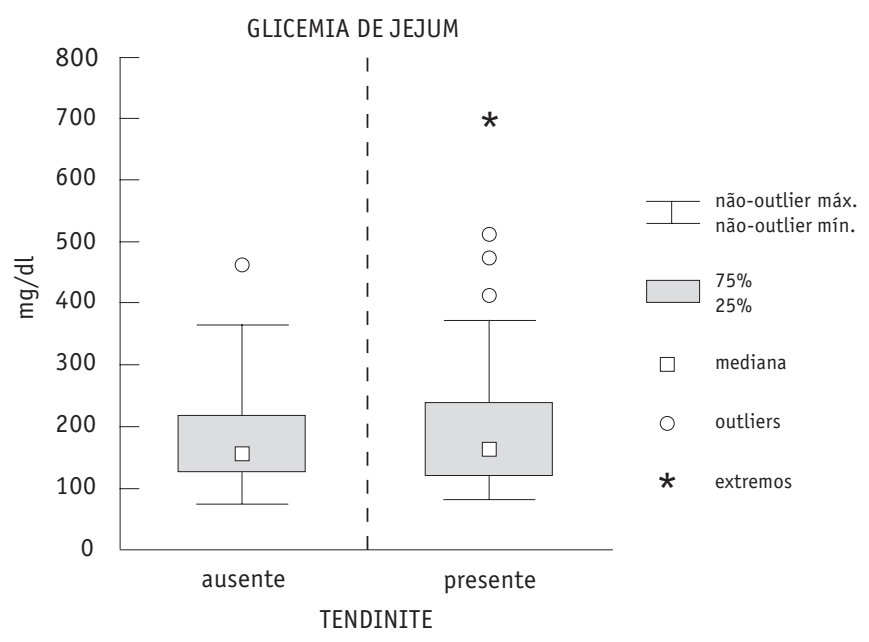

Figura 3 - Comportamento da glicemia de jejum nos pacientes com e sem tendinites.

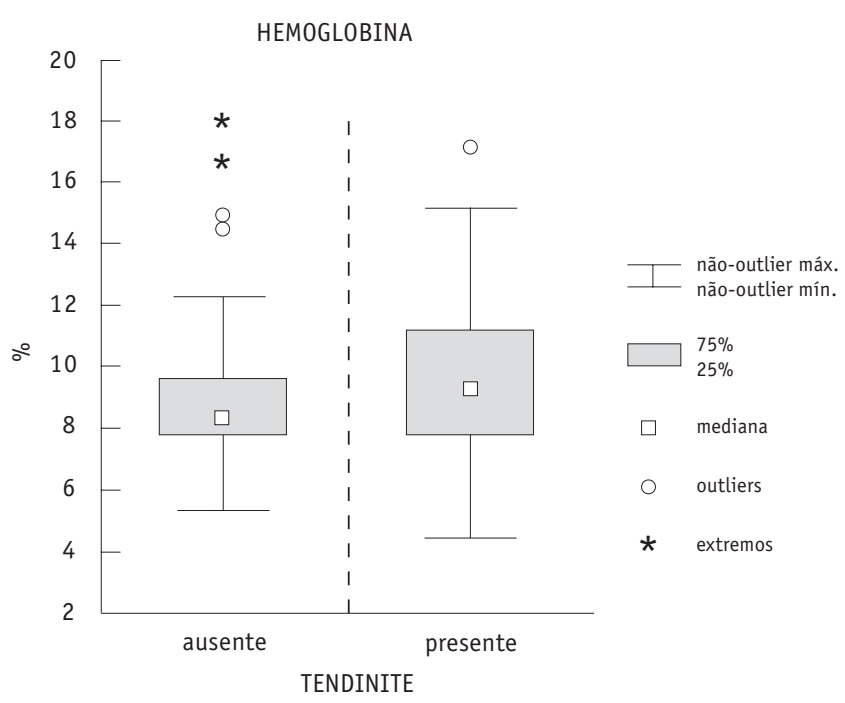

Figura 4 - Comportamento da hemoglobina $A_{1 C}$ nos pacientes com e sem tendinites.

No que concerne à possível associação da taxa de glicemia de jejum e ocorrência de tendinites, apesar de os diabéticos com achados positivos apresentarem uma média de glicemia levemente superior à dos sem achados positivos, o estudo pelo método de Mann Whitney não demonstrou uma correlação significativa $(p=0,110)$. O mesmo aconteceu em relação à $\mathrm{Hb} \mathrm{A} 1 \mathrm{C}$; diabéticos com achados positivos apresentavam uma média de $\mathrm{Hb} \mathrm{A} 1 \mathrm{C}$ levemente superior (tabela 3), mas o teste de Mann Whitney não aponta para diferença significativa $(\mathrm{p}=0,093)$. 
TABELA 3

DESCRIÇÃO DA TAXA MÉDIA DE GLICEMIA EM JEJUM E COM RELACÃO ÀS TENDINITES

\begin{tabular}{lcc}
\hline Grupos & $\begin{array}{c}\text { Glicemia em jejum } \\
\text { (médias) }(\mathrm{em} \mathrm{mg} / \mathrm{dl})\end{array}$ & $\begin{array}{c}\text { Hemoglobina } \mathrm{A}_{\mathrm{c}} \\
\text { média }(\mathrm{em} \%)\end{array}$ \\
\hline Sem tendinites $(\mathrm{n}=100)$ & $177,23(\mathrm{DP} \pm 78,67)$ & $9,03(\mathrm{DP} \pm 2,08)$ \\
Com tendinites $(\mathrm{n}=46)$ & $210,78(\mathrm{DP} \pm 120,18)$ & $9,58(\mathrm{DP} \pm 2,42)$ \\
\hline
\end{tabular}

${ }^{*} \mathrm{n}=$ número de pacientes da amostra; $\mathrm{DP}=$ desvio-padrão

Estudando-se a ocorrência de tendinites e/ou entesopatias em relação à idade dos pacientes pelo teste de Mann Whitney, observou-se uma ausência de correlação ( $p=$ $0,26)$. O mesmo aconteceu com a presença de tendinites e/ou entesopatias e o tempo de doença (teste do Quiquadrado; $\mathrm{p}=0,267)$. $\mathrm{O}$ tipo de diabetes também não influiu no aparecimento de tendinites e/ou entesopatias (teste exato de Fisher; $\mathrm{p}=0,33$ ), nem se encontrou alguma correlação com o tipo de tratamento utilizado (Quiquadrado; $\mathrm{p}=0,252)$ (Tabela 4).

TABELA 4

RELAÇÃO ENTRE O TIPO DE DIABETES E DE TRATAMENTO E A OCORRÊNCIA DE TENDINITES

\begin{tabular}{l|cc|ccc}
\hline & \multicolumn{2}{|c|}{ Tipo de diabetes } & \multicolumn{3}{|c}{ Tipo de tratamento } \\
& Tipo 1 & Tipo 2 & Dieta & H. oral & Insulina \\
& $(n=12)$ & $(n=137)$ & $(n=19)$ & $(n=77)$ & $(n=53)$ \\
\hline Com tendinite & $n=5$ & $n=94$ & $n=3$ & $n=26$ & $n=19$ \\
Sem tendinite & $n=7$ & $n=43$ & $n=16$ & $n=51$ & $n=34$ \\
\hline
\end{tabular}

$\mathrm{H}=$ hipoglicemiante; $\mathrm{n}=$ número de pacientes da amostra

\section{DISCUSSÃO}

Hiperglicemia crônica é responsável por uma série de transformações no colágeno muito semelhante àquelas observadas no envelhecimento ${ }^{(2,4)}$. Essas reações são chamadas genericamente de reações de Maillard e acontecem da seguinte maneira:

1. Em um primeiro momento, existe uma glicolização não enzimática dos agrupamentos amina das proteínas que contém resíduos de lisina. Essa ligação é inicialmente reversível, mas evolui rapidamente para a formação de um produto mais estável, conhecido como produto de Amadori;
2. O produto de Amadori sofre desidratação, oxidação, divisão e um rearranjo, formando os chamados produtos finais de glicolização avançada (AGEs - advanced glycation end products);

3. Os AGEs são considerados os elementos responsáveis por uma acentuação no cross linking do colágeno, com conseqüente aumento na sua rigidez, na resistência à digestão por enzimas e de sua fluorescência. Promovem, também, alterações na cor do tecido, o qual fica mais amarronzado (Figura 5).

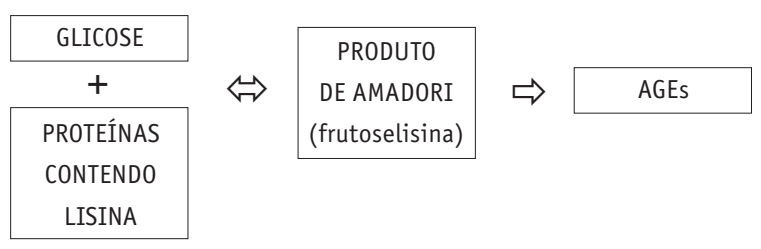

Figura 5 - Reação de Maillard.

Os AGEs são encontrados no cristalino, no colágeno, na hemoglobina, estão ligados à beta- 2 microglobulina e mielina ${ }^{(4)}$. Sua produção é proporcional ao tempo de hiperglicemia $^{(4)}$ e está relacionada de maneira positiva com a retinopatia e a nefropatia do diabético ${ }^{(5)}$. Além disso, esses AGEs são capazes de aprisionar proteínas como a LDL (lipoproteína de baixa densidade) permitindo o seu acúmulo (por diminuição de metabolização), além de promover a indução de quimiotaxia transendotelial dos monócitos, secreção do fator de crescimento derivado de plaquetas e citocinas (como a interleucina $1 \mathrm{e}$ o fator de necrose tumoral) ${ }^{(6)}$.

A síndrome da mobilidade articular diminuída é observada em um grande número de pacientes diabéticos e tem sido detectada em mãos ${ }^{(7)}$, pés ${ }^{(7)}$, coxo-femorais, ombros ${ }^{(7-}$ ${ }^{10)}$ etc., sendo atribuída às alterações do colágeno já descritas. Segundo Monnier et al., o grau de fluorescência do colágeno obtido da pele de diabéticos está relacionado à severidade da retinopatia, da lesão de parede arterial, e com o grau de rigidez da mão ${ }^{(5)}$. Acredita-se que as tendinites e as entesopatias possam estar ligadas ao mesmo mecanismo fisiopatológico em tecido colagenoso peritendíneo ${ }^{(11)}$.

No presente estudo pudemos verificar uma alta prevalência de tendinites e/ou entesopatias em pacientes diabéticos, as quais envolviam principalmente estruturas de articulações de membro superior.

Não se conseguiu demonstrar nenhuma associação entre a ocorrência de glicemia de jejum, as dosagens de $\mathrm{Hb}$ 
A1C e a ocorrência de tendinites e entesopatias. A falta de correlação com níveis de glicemia de jejum é facilmente entendida, haja vista a fugacidade desse valor. Como explicar, então, a falta de correlação com $\mathrm{Hb} \mathrm{A} 1 \mathrm{C}$, uma vez que esta se forma por um mecanismo semelhante ao já descrito de glicolização não enzimática?

Uma das hipóteses que podem ser levantadas para essa aparente discrepância é a de que o processo que acontece nos tendões e nas enteses é muito mais lento do que o que acontece com a hemoglobina. Aliás, isso já foi constatado em relação à pele. A concentração de $\mathrm{Hb} \mathrm{A} 1 \mathrm{C}$ reflete a concentração da glicose média nas 6 a 8 semanas prévias à sua dosagem ${ }^{(12)}$. Na pele, um controle rígido da glicemia leva cerca de 4 meses para reverter os efeitos da reação de Maillard ${ }^{(13)}$. Isso acontece porque, no sangue, a concentração da $\mathrm{Hb}$ A1C está influenciada pela sobrevida das células vermelhas ${ }^{(12)}$, que é pequena.

Uma outra hipótese a ser contemplada é a possibilidade de que exista influência de um fator genético, conforme sugerido por Silverstein et al. ${ }^{(14)}$.

\section{REFERÊNCIAS}

1. Rosenbloom AL, Silversteim JH: Connective tissue and joint disease in diabetes mellitus. Endocrinol Metabol Clin 25(2): 473-83, 1996.

2. Monnier V, Sell DR, Abdul-Karim F, Emancipator SN: Collagen browning and cross-linking are increased in chronic experimental hyperglicemia. Diabetes 37: 867-72, 1988.

3. Sheon RP, Moskowitz RW, Goldberg VM: Soft Tissue Rheumatic Pain: recognition, management, prevention. Philadelphia: Lea \& Ferbiger, 302, 1982.

4. Raj DSC, Choudhury D, Welbourne TC, Levi M: Advanced glycation and products: a nephrologist's pespective. Am J Kidney Dis 35(3): 365-78, 2000 .

5. Monnier VM, Bautista O, Kenny D, et al: Skin collagen glycation, glycoxidation and crosslinking are lower in subjects with long term intensive versus conventional therapy of type 1 diabetes. Relevance of glycated collagen products versus $\mathrm{Hb}$ A1c as markers of diabetic complications. Diabetes 48: 870-80, 1999.

6. Isdale $\mathrm{AH}$ : The $\mathrm{ABC}$ of the diabetic hand: advanced glycosilation and products, browning and collagen. Br J Rheumatol 32(10): 559 61, 1993.

7. Garg SK, Chase HP, Marshall G, Jackson WE, Holmer D, Hoops S, Harris S: Limited joint mobility in subjects with insulin dependent diabetes mellitus: relationship with eye and kidney complications. Arch Dis Child 67: 96-9, 1992
Dessa maneira, quando o clínico se pergunta sobre os efeitos da melhoria do controle glicêmico sobre a tendinite, há que se lembrar de que este não se fará a curto prazo, exigindo outras formas de tratamento até que o processo etiopatogênico possa ser revertido ou melhorado.

Por outro lado, é interessante notar a prevalência quase igual de entesopatias e tendinites em homens e mulheres diabéticas quando é fato sabido que, na população em geral, esta é uma patologia mais comum em mulheres, uma vez que elas têm uma maior mobilidade articular, estando, portanto, mais sujeitas a microtraumatismos ${ }^{(15)}$.

Tendinites e entesopatias em pacientes diabéticos podem oferecer dificuldades de tratamento, uma vez que o uso de infiltrações com corticóide pode prejudicar o controle glicêmico, e, muitas vezes, os antiinflamatórios nãohormonais estão contra-indicados pela existência de comorbidades renais e cardíacas muito comuns nesses pacientes. Maiores estudos são necessários nessa área, principalmente no sentido de esclarecer o tempo necessário de controle glicêmico para reversão do processo.

8. Morén-Hybbinette I, Moritz U, Scherstén B: The painful diabetic shoulder. Acta Med Scand 219: 507-14, 1986.

9. Sattar MA, Luqman WA: Periarthritis: another duration related complications of diabetes mellitus. Diabetes Care 8(5): 507-10, 1985.

10. Siegel L, Cohen NJ, Gall EP: Adhesive capsulitis: a sticky issue. Am Fam Phys, 59(7): 1843-52, 1999.

11. Blyth MJC, Ross DJ: Diabetes and trigger finger. J Hand Surg (Br) 21B(2): 244-5, 1996.

12. McCulloch DK: Estimation of blood glucose control in diabetes mellitus. In: Rose B (ed.): Up to date.com versão 9.2, feb 13, 2001.

13. Lyons TJ, Bailie KE, Dyer DG, Dunn JA, Baynes JW: Decrease in skin collagen glycation with improved glycemic control in patients with insulin-dependent diabetes mellitus. J Clin Invest 87: 1910-5, 1991.

14. Silverstein JH, Gordon G, Pollok BH, Rosembloom AL: Long term glycemic control influences the onset of limited joint mobility in type 1 diabetes. J Pediatr 132(6): 944-7, 1998.

15. Machtey I: Tendinitis and abnormal glucose tolerance (letter). Scand J Rheumatol 15: 224, 1986. 